\title{
The use and context of the term "multimorbidity" in rheumatoid arthritis: a systematic literature review
}

Mrinalini Dey ${ }^{1,2}$, Amanda Busby ${ }^{3}$, Helen Elwell ${ }^{4}$, Arthur Pratt ${ }^{5,6}$, Adam Young $^{3}$, John Isaacs ${ }^{5,6}$, Elena Nikiphorou ${ }^{7}$

${ }^{1}$ Institute of Life Course and Medical Sciences, University of Liverpool, Brownlow Hill, Liverpool, L69 3BX, UK

${ }^{2}$ Department of Rheumatology, Aintree Hospital, Liverpool University Hospitals NHS Foundation Trust, Lower Lane, L9 7AL, UK

${ }^{3}$ Centre for Health Services and Clinical Research, Life and Medical Sciences, University of Hertfordshire, College Lane, Hatfield, AL10 9AB, UK

${ }^{4}$ BMA Library, British Medical Association, BMA House, Tavistock Square, London, WC1H 9JP

${ }^{5}$ Newcastle University Translational and Clinical Research Institute, Faculty of Medical Sciences, Framlington Place, Newcastle upon Tyne, NE2 4HH

${ }^{6}$ Musculoskeletal Unit, Newcastle upon Tyne Hospitals NHS Foundation Trust, Freeman Hospital, Freeman Road, Newcastle upon Tyne, NE7 7DN

${ }^{7}$ Centre for Rheumatic Diseases, King's College London, London, UK

Corresponding author: Dr Mrinalini Dey, Institute of Life Course \& Medical Sciences, Department of Rheumatology, $3^{\text {rd }}$ Floor Clinical Sciences Centre, Aintree Hospital, Liverpool University Hospitals NHS Foundation Trust, Lower Lane, L9 7AL, UK.

Mrinalini.dey@nhs.net

Word count: 3482 


\begin{abstract}
Objectives

This systematic literature review (SLR) aimed to determine term(s) used to describe co-existing conditions in rheumatoid arthritis (RA) literature, to inform the need for an operationalised definition of multimorbidity in rheumatic diseases.
\end{abstract}

\title{
Methods
}

An SLR was undertaken to establish how "multimorbidity" and related terms are used in RA literature. We searched Medline, Embase, Health Technology Assessment and Cochrane Databases for articles discussing RA with multimorbidity. There was no time restriction, with articles published between 1946 and $11^{\text {th }}$ August 2020 (search date) included. There was no restriction on language or study type. The primary outcome was use and/or definition of "multimorbidity" in RA. Information extracted included: term(s) used to define co-existing conditions and definition (if included); use of comorbidity/multimorbidity score; co-existing conditions studied; use of term "index disease" to describe RA (more applicable to comorbidity, than multimorbidity).

\section{Results}

354 articles were initially identified, 39 meeting inclusion criteria. Eight articles used the term "multimorbidity"; 18 used "comorbidity." 12 articles used both terms, seven synonymously. One used no term. 14 articles fully defined their term and four provided partial definitions. The number of co-existing conditions ranged from one to 121. Twelve articles used a comorbidity/multimorbidity score; the term "index disease" was used to refer to RA in four articles.

\section{Conclusions}

Our results demonstrate inconsistent use of the term multimorbidity, with variation in reported numbers of comorbidities. There is a need for improved multimorbidity assessment in RA, including operationalised use and definition tailored to the multi-facetted nature of these patients.

Keywords: multimorbidity, comorbidity, rheumatoid arthritis, rheumatic diseases

\section{Key messages:}

1. No operationalised definition exists for multimorbidity in rheumatic diseases, causing inconsistent use of the term.

2. The number of comorbidities included within any definition of multimorbidity varies between studies.

3. A better understanding and use of "multimorbidity" may encourage more holistic patient care.

\section{Introduction}

Multimorbidity is becoming increasingly common in patients with rheumatic and musculoskeletal conditions (RMDs) $(1,2)$. There have been several attempts to define multimorbidity as a concept. The Academy of Medical Sciences, Medical Research Council and National Institute for Health 
Research define multimorbidity as "the co-existence of two or more chronic conditions, each one of which is either: a physical non-communicable disease of long duration, such as a cardiovascular disease or cancer; a mental health condition of long duration, such as a mood disorder or dementia; an infectious disease of long duration, such as HIV or hepatitis C (3). The National Institute for Clinical Excellence defines multimorbidity as "two or more long-term health conditions, which can include: defined physical or mental health conditions, such as diabetes or schizophrenia; ongoing conditions, such as learning disability; symptom complexes, such as frailty or chronic pain; sensory impairment, such as sight or hearing loss; alcohol or substance misuse" (4). A consensus definition, particularly across the rheumatic diseases, is lacking, with inconsistencies in the usage of the term.

As a concept, multimorbidity has only recently started to come to the attention of the healthcare community. Meanwhile, the related concept of 'comorbidity', defined as "the existence or occurrence of any distinct additional entity during the clinical course of a patient who has the index disease under study" (5), has long been explored in RMDs, represented by the vast amount of literature that addresses this issue.

In rheumatoid arthritis (RA) in particular, comorbidities have been studied in enormous depth. Comorbidity burden, particularly at presentation, has increased markedly over the last 25 years, with some of the most common being obesity and lung pathologies such as chronic obstructive pulmonary disease (COPD). In the case of COPD, for example, this is a key predictor of early mortality in early RA (6-8). The clinical burden of multimorbidity is becoming increasingly recognised in reallife practice, but the number of studies exploring multimorbidity have failed to match the scale of this (9). Given the chronic nature of RA, the potential extra-articular complications, associations with other chronic conditions including cardiovascular disease, and psychological and quality of life factors, it is increasingly important to improve our understanding of multimorbidity in RA, in order to enhance patient management, encourage a more patient-centric approach to care, and improve research practices in this area (2).

Whilst subtle differences exist between comorbidity and multimorbidity, the two are not mutually exclusive (Table 1; Figure 1). Nonetheless, it has previously been noted that the terms are often used, inappropriately, interchangeably in clinical and research literature, particularly as the research interest in this area has increased over the past two decades $(9,10)$. An important difference between the two terms is that, while comorbidity refers to an index disease with a co-existing condition or conditions, multimorbidity considers the patient as a whole taking into account all biological, psychological, and social factors integral to their care. Thus, the use of the term multimorbidity inherently suggests a more holistic approach to care, with 'comorbidity' bringing the emphasis back to an index disease, with other conditions surrounding this. The question is, where is the patient placed in the latter approach? In the context of an ageing population and the paradigm chronic rheumatic disease, RA, addressing multimorbidity is likely to improve the holistic management of patients.

One of the main barriers to the appropriate use and understanding of multimorbidity as a concept in RA is the lack of operationalised definition, for both clinical and research purposes. The aims of this systematic review were therefore three-fold:

- to determine the collective term(s) used to describe the presence of co-existing conditions in the context of RA in literature;

- to describe the various types of scores or indices used to quantify the presence and impact of co-existing conditions, and the frequency and context of their use. 
- to determine the frequency and context of use of the term "index disease," with reference to RA as the main disease of interest.

\section{Methods}

This systematic literature review (SLR) was conducted in accordance with the Cochrane Handbook (11) and reported as per the Preferred Reporting Items for Systematic Reviews and Meta-Analyses (PRISMA) guidelines (12).

The protocol was developed by EN, MD and HE, and registered in the Research Registry database of systematic reviews (13). The search question was established as: How is the term 'multimorbidity', and related terms, used in the context of rheumatoid arthritis (RA) in the clinical and research literature? This question was framed and structured using the 'Population, Phenomenon of interest and Context' (PICo) format (14).

\section{Population}

Our population are patients with RA. Therefore, a study was included if participants included subjects with RA. Studies in which RA was recorded as a co-existing condition, with another condition as the index (i.e. main) disease, were excluded.

\section{Phenomenon of interest}

The phenomenon of interest was multimorbidity (or similar MeSH terms: multimorbid\$ or multimorbid\$ or multiple comorbid\$ or multiple co-morbid\$ or multi-comorbid\$ or multi-co-morbid\$ or multiple morbidit or polymorbid\$ or poly-morbid\$, or multiple or co-existing conditions; full search strategy available in supplementary material).

\section{Context}

Articles retrieved were those including the above participants and phenomenon of interest, in any context. The search was kept deliberately broad, and authors manually extracted articles relevant at the data selection stage. This ensured all articles featuring a definition or outline of their understanding of multimorbidity were identified.

\section{Search strategy, databases searched, and study selection}

The search strategy for this SLR was developed by two authors (MD and EN) with the help of an expert librarian in undertaking SLRs and clinical research (HE). The combination of keywords used in this search and full search strategy is available in the supplementary material. Our initial search had no restriction on language or article type. The bibliographic databases Medline, Embase, International Network of Agencies for Health Technology Assessment (INAHTA) and Cochrane Databases (Systematic Reviews, Register of Controlled Clinical Trials, Methodology Register) were searched. Conference abstracts and articles in languages other than English were screened separately. The search conducted had no time restriction and included articles in the databases searched between 1946 and the $11^{\text {th }}$ of August 2020, when the search was performed. 
There were no restrictions on the types of studies to be included in the review. For example, observational (including prevalence studies, cross-sectional), interventional (longitudinal, randomised controlled trials), qualitative studies, commentaries, policy documents or reviews, were all included.

An initial scoping review was performed, to search for both comorbidity- and multimorbidity-related papers in RA, and review those considered relevant, including guidelines, recommendations, and other key papers on the topic. This initial scoping search yielded almost 15,000 articles. On screening 200 of these articles, it was clear that most articles focussed on comorbidity, rather than multimorbidity.

Ultimately, the focus of the review was on "multimorbidity", the co-existence of multiple health conditions, as opposed to "comorbidity" in view of the research question under study. Specifically, we reviewed the definitions used in the existing literature relating to multimorbidity in the context of RA, acknowledging (from the initial scoping review) that while thousands of comorbidity papers exist, far fewer use and define multimorbidity in a consistent manner. An overview of the results of the initial scoping review is available in the supplementary material.

All identified full-length articles were uploaded into EndNote VX9 (Clarivate Analytics, PA, USA), with duplicates subsequently removed (Figure 2 ). Titles and abstracts were screened by MD, to assess eligibility. The full articles which met the inclusion criteria were then examined in detail by MD. As a validation exercise, $20 \%$ of the articles were screened at the abstract and full paper stage by a second author, $A B$. Any disagreements between reviewers were resolved through discussion and with input from a third reviewer (EN).

\section{Assessment of risk of bias, data extraction, and synthesis}

Risk of bias in each included study was assessed, using the appropriate tool for the article in question. Details of tools used, along with results for each article, are provided in the supplementary material. Narrative literature reviews and editorials were excluded from risk of bias assessment. Data extraction from the included articles was undertaken by MD, with $20 \%$ of articles extracted by AB. No papers, or additional data or supplementary material was required from authors.

For each selected article, in addition to basic information, the following information was extracted: main disease of interest; the use of the term "index disease" to describe the disease of interest (i.e. $\mathrm{RA}$ ); the use of a comorbidity/multimorbidity score and the type if applicable; co-existing conditions under study; term used to define the presence of co-existing conditions and its definition (where included in the article); whether RA was included as part of the multimorbidity definition; the switch between terms and definitions within the body of the article. We examined the term "index disease" to identify variations and heterogeneity in its use within the literature. "Index disease" in the wider sense is more applicable to comorbidity, as opposed to multimorbidity in which all patient- and disease-related factors are given equal attention (Figure 1).

\section{Results}

A total of 354 articles were identified through the initial search strategy. On exclusion of those which were in languages other than English, this was reduced to 326. After deduplication, 230 articles were included in the review. 191 records were excluded on screening the abstracts, including those in 
which RA was described as a co-existing condition, and not the main disease of interest (i.e. the index disease). Ultimately, 39 full-text articles were screened for eligibility, with no records excluded at this stage. At both the abstract and full-text stage, $100 \%$ concordance was achieved between the two reviewers ( $M D$ and $A B$ ) on $20 \%$ validation check. Therefore, all 39 articles were deemed eligible for data extraction. Figure 2 summarises the article numbers from 'start to end' of the article retrieval process.

In addition to the strategy adopted, for further validation, reference lists for included articles were also screened, although this did not yield further eligible records.

The types of articles included in this review were: observational cohort $(n=13)$, case-control $(n=7)$, cross-sectional $(n=6), \operatorname{SLR}(n=3)$, scoping literature review $(n=4)$, protocol for $\operatorname{SLR}(n=1)$, protocol for randomised controlled trial $(R C T)(n=1)$, editorial $(n=2)$, audit $(n=1)$ and consensus statement for practice $(n=1)$. The main characteristics of each of these articles are summarised in Table 2 . In the case of the protocols for the SLR and RCT, further publications from these projects were not found on further searching and were therefore unavailable for review $(15,16)$.

128 conference abstracts were identified in our search strategy. On screening, 54 articles met the eligibility criteria. A summary of included abstracts is available in the supplementary material. Of the 54 abstracts, six were identified in our search of full-length articles. With regards definitions of the term(s) used to define co-existing conditions, three of the 54 abstracts defined the term(s) either in part or full. Of the 54 abstracts, 12 stated a priori the number of co-existing conditions under study, while 15 used one or more comorbidity or multimorbidity score or index as part of their study.

\section{Term(s) used to define the presence of co-existing conditions}

There were differences between articles with regards the terms used to define the presence of coexisting conditions, with results summarised in Table 3. Terms used included "comorbidity" (or "comorbidity"), "multimorbidity" or a combination of the two words.

The term "comorbidity" or "co-morbidity" was used in 18 of the 39 articles, while eight used "multimorbidity". One article had no term to describe co-existing conditions. A combination of both "multimorbidity" and "comorbidity" was used in twelve of the 39 articles. In five of these 12 papers, the two terms were used appropriately (i.e. not synonymously), with adequate definitions provided within the text. In the remaining seven articles, the terms "comorbidity" and "multimorbidity" were inappropriately used synonymously, with six of these seven articles failing to provide a complete definition of the terms used.

Of the 39 included articles, 14 provided definitions of the term used to describe the presence of coexisting conditions, with a further four providing a partial definition. Where the term "comorbidity" was used, three of the 18 articles provided a definition. Where "multimorbidity" was used, six of the eight articles gave a definition, with one giving a partial definition (Table 3).

The number of co-existing conditions considered as comorbidities and/or multimorbidity ranged from one to 121 (in 15 defined clusters), with five articles failing to define the type or number of coexisting conditions within the main text. The level of detail provided on these co-existing conditions also varied between papers, e.g. whilst some stated "cardiovascular disease" as a standalone condition, others further refined this to include conditions such as "arrhythmia," "previous myocardial infarction," and "angina," as conditions in their own right.

Co-existing conditions were also checked for their inclusion of RA, as part of the comorbidity or multimorbidity term. RA was defined as a co-existing condition in two articles, justified in the text 
due to study of extra-articular features (17), and the co-existence of psoriatic arthritis with RA (18). Two further articles failed to make it clear as to whether RA was included in their definition of coexisting conditions; also neither listed the co-existing conditions under study $(19,20)$.

In one article, no term was used to define the presence of co-existing conditions in the context of RA (21). This article, in the form of a letter, reported results from an observational study used to evaluate the impact of the 2015/2016 EULAR recommendations on cardiovascular disease in the context of RA. Whilst reference was made to co-existing cardiovascular conditions throughout the text, no single word was used to define the presence of these in the context of RA.

\section{Use of a multimorbidity or comorbidity index score}

Out of the 39 included articles, 12 (comprising cohort, cross-sectional, and case-control studies) stated the use of a multimorbidity or comorbidity index in their article (Table 4). Indices used were: Charlson Comorbidity Index ( $n=1)$; Counted Multimorbidity Index $(n=3)$; Elixhauser Comorbidity Index ( $n=2)$; Malmo Classification of extra-articular features ( $n=1)$; Multimorbidity Index (using counted and weighted indices) ( $n=3)$; Weighted Multimorbidity Index $(n=1)$; Rheumatic Disease Comorbidity Index $(n=1)$. Review articles and editorial articles were not assessed for their use of a comorbidity index, due to discussion of multiple papers featuring different (or no) indices.

The context in which a comorbidity or multimorbidity score was used is summarised for each of these 12 papers in Table 4.

Of note, articles using the same comorbidity index scores did not necessarily record the same coexisting conditions. A greater degree of variation was observed in type and number of co-existing conditions in the articles which had not used a score for this purpose.

\section{Description of disease of interest as the "index" disease}

Out of the 39 articles, 26 named RA as the main disease of interest under study or discussion in the main text. In three of the included articles, RA was described as one of two main diseases of interest; the other diseases of interest in each of the three papers, respectively, were osteoarthritis (20), psoriatic arthritis (18), and antiphospholipid syndrome (22). In ten of the 39 articles, the conditions of interest were described under the grouped term "rheumatic diseases" (or similar), with RA named as one of the diseases of interest.

The disease of interest (RA) was referred to as the "index" disease in four of the 39 articles. Of note, three of these were by the same first author, whilst the fourth was an editorial (2,23-25). All four articles also had appropriate use and definition of the term "multimorbidity".

\section{Discussion}

This systematic review addresses the concepts of multimorbidity, the co-existence of multiple conditions and/or multiple comorbidities, in the context of RA, as currently used in the existing literature. To our knowledge, this is the first in-depth attempt to review and understand the usage and meaning of "multimorbidity" in RA.

\section{Term(s) used to define the presence of co-existing conditions}

There is marked variation in the terminology used to describe the presence of multiple co-existing conditions in literature on RA, and also within individual articles, sometimes with no consistency of use or switching between terms. Often, no definition or reference is provided for the term used. 
Where the term "multimorbidity" is used, it is more likely to be defined in the text, compared to the term "comorbidity." This is seen most obviously when both terms are used, but only "multimorbidity" is defined (16,26-29). However, despite multimorbidity being defined in the text more commonly than comorbidity, this does not always lead to consistent usage throughout the article. This perhaps indicates a need for an operationalised definition for the two terms and greater clarity in differentiation between the two.

This review also found there to be little consistency in the number or type of co-existing conditions which constitute "multimorbidity". The largest number of co-existing conditions under study in a single paper was 121 (30). Multimorbidity encompasses chronic, as opposed to co-existing acute, conditions, but this is not always the case in literature discussing multimorbidity (2). A potential solution to this, in addition to an operationalised definition, may be the use of a graded multimorbidity score in RMDs. This would reflect the fact that there is no one index condition, but also that the clinical state and potential management is different between patients with varying numbers of co-existing conditions.

\section{Use of a multimorbidity or comorbidity index score}

This SLR demonstrates that a variety of multimorbidity or comorbidity index scores are in use in studies on RA. Of note, there is no consistency in the use of a score in a certain type of study. Some, such as the Charlson Index, are not specific to RA, or even RMDs, which renders them less useful in this context and, in some cases, leading authors to adapt the scores to suit the needs of the research. The number and type of co-existing conditions recorded in a score are also not necessarily the same between studies using the same score.

This further reinforces the need for an operationalised definition of "multimorbidity" which encompasses the patient as a whole and, if appropriate, a defined set of conditions. If such a score is to be applied more broadly to RMDs, it should account for the fact that one RMD (e.g. RA) may be the index disease in one study, and a co-existing condition in another. It is also important to note that, while it is important in some circumstances to consider the primary, or index disease and the co-existing conditions alongside this, it is becoming increasingly important to embrace a more holistic approach to management. This requires an "overview" approach to the patient, rather than considering diseases as separate entities.

\section{Description of disease of interest as the "index" disease}

The term, "index disease", which defines the disease of interest in a given article, is rarely used. This is more likely due to a lack of understanding and awareness of its use, rather than a need to avoid this term when discussing multimorbidity. In order to use the term "multimorbidity" in a consistent manner across RA literature, it is important to appreciate the symbiosis of multiple conditions that exist in such a patient, a common clinical state across the RMDs, as opposed to existence of a single index disease and secondary conditions 'surrounding' it.

A limitation of this review is that $20 \%$ of the initially retrieved articles were screened by two reviewers. Given the complexity of the review question and topic under study, every effort was made to ensure reviewers were as thorough as possible. Subsequently, no discrepancies were identified between reviewers. The strong agreement in the screened random sample by the two reviewers was reassuring.

\section{Conclusion}


Multimorbidity has been discussed in the literature for many years, and in many areas of medicine. Yet, there remains no single definition or method of assessment for multimorbidity in RMDs, which by their definition encompass an array of co-existing conditions within a single patient (9). This does not simply refer to multiple conditions in a given person, but also factors such as iatrogenic disease and polypharmacy (Figure 1b). Multimorbidity implies that each patient's management, and clinical outcomes, are assessed as a whole. To this end, health-related quality of life (HRQoL) has previously been suggested as a baseline from which to build a multimorbidity scoring tool (31). However, HRQoL assessments and other tools and scoring systems before these, have failed to be embraced by the rheumatology community, for either clinical or research purposes.

The results of this SLR have highlighted several areas for improvement in the area of multimorbidity assessment in RA, and RMDs more widely. There is a need for more robust and streamlined assessment of multimorbidity in RA, including an operationalised use and definition, and perhaps also a score or index tailored to the multi-facetted nature of patients with such chronic diseases. These would improve consistency across clinical practice and ultimately lead to more patient-centric and holistic patient management.

The authors declare no conflicts of interest.

$M D, E N$ and $H E$ devised the search strategy and protocol for the systematic review. MD and $A B$ conducted the screening and data extraction. MD drafted the initial manuscript which was expanded and edited by all co-authors. All authors read and approved the final manuscript.

\section{Funding.}

MD is an NIHR-funded Academic Clinical Fellow.

JDI is a NIHR Senior Investigator and his work is supported by the NIHR Newcastle Biomedical Research Centre and the Research Into Inflammatory Arthritis Centre Versus Arthritis. The views expressed are those of the authors and not necessarily those of the NHS, the NIHR or the Department of Health and Social Care.

This work was conducted as part of the BMA Doris Hillier Award received by EN.

Ethical approval was not applicable to this work.

All data collected as part of this systematic review is available in the supplementary material and also obtainable upon request. 


\begin{tabular}{|c|c|c|}
\hline Concept & Multimorbidity & Comorbidity \\
\hline Focus & $\begin{array}{l}\text { No index disease defined, patient- } \\
\text { centred concept }\end{array}$ & $\begin{array}{l}\text { Index disease as centre of interest, co-existing } \\
\text { conditions considered as additional entities }\end{array}$ \\
\hline $\begin{array}{l}\text { Importance of } \\
\text { conditions }\end{array}$ & All conditions considered equally & $\begin{array}{l}\text { Different importance of conditions, i.e. primary } \\
\text { index disease, and secondary co-existing } \\
\text { conditions }\end{array}$ \\
\hline $\begin{array}{l}\text { Interaction of } \\
\text { conditions }\end{array}$ & Co-existing conditions interact & $\begin{array}{l}\text { Co-existing conditions interact with index } \\
\text { disease, but not necessarily with one another }\end{array}$ \\
\hline Conditions included & Chronic conditions & Any co-existing condition (i.e. acute and chronic) \\
\hline Definition & $\begin{array}{l}\text { Concept considered a standalone } \\
\text { entity; not the sum of many parts or } \\
\text { a key central condition }\end{array}$ & $\begin{array}{l}\text { Cluster of single conditions surrounding an index } \\
\text { disease }\end{array}$ \\
\hline
\end{tabular}

Table 1: Key differences between multimorbidity and comorbidity (Adapted from Radner, H., Yoshida, K. and Smolen, J. S. (2014) 'Multimorbidity and rheumatic conditions - Enhancing the concept of comorbidity', Nature Reviews Rheumatology.) 


\begin{tabular}{|c|c|c|}
\hline Author/Year & Country & Summary of article aims and context of use of comorbidity or multimorbidity terms \\
\hline \multicolumn{3}{|l|}{ Observational cohort $(n=13)$} \\
\hline Armagan $2018(32)$ & Turkey & $\begin{array}{l}\text { Assessment of frequency of comorbidities and multimorbidities in RA patients under biologic therapy and their effects on biological DMARD choice, timing, } \\
\text { and response. }\end{array}$ \\
\hline Biggioggero 2019 (33) & Italy & $\begin{array}{l}\text { Retrospective evaluation of impact of comorbidities on treatment choice, clinical response, and retention rate in patients with RA treated with TNFi, using } \\
\text { the RDCI. }\end{array}$ \\
\hline Bili 2011 (34) & USA & $\begin{array}{l}\text { To determine if RA patients receive equal recommended preventive services in RA-related testing (dyslipidemia, osteoporosis) and in non-RA-related } \\
\text { testing (mammography, cervical cancer), compared to general population, in the context of increased CVD comorbidity risk in RA. }\end{array}$ \\
\hline Chen 2019 (18) & Taiwan & $\begin{array}{l}\text { To determine the prevalence and the clinical features of RA among patients with psoriasis in a tertiary referral centre, including the presence of } \\
\text { comorbidities. }\end{array}$ \\
\hline Crilly 2014 (17) & UK & Quantification of relative contribution of extra-articular features and comorbidities to quality of life in patients with RA. \\
\hline Daïen 2018 (21) & France & $\begin{array}{l}\text { Evaluation of the impact of the updated 2015/2016 EULAR CVD recommendations as compared with the } 2009 \text { recommendations on CVD risk stratification } \\
\text { and statin indication in a prospective cohort of RA patients, in the context of increased CVD comorbidity risk in RA. }\end{array}$ \\
\hline Gil-Conesa 2020 (19) & Spain & $\begin{array}{l}\text { Estimation of rates of hospitalisation of RA in Spain, when RA is the main cause of hospitalisation or a comorbidity, and describe the main diagnosis when } \\
\text { RA was not the cause of hospitalisation, and comorbidities when it was the main cause. }\end{array}$ \\
\hline Hope 2020 (35) & UK & $\begin{array}{l}\text { Comorbidities studied as part of investigation into rates of non-adherence to methotrexate after therapy commencement and identifying patient } \\
\text { characteristics prior to start of drug to predict non-adherence. }\end{array}$ \\
\hline Radner 2015 (1) (23) & Austria/USA & Assessment of the effect of multimorbidity status on treatment effects in RA patients initiating any DMARD therapy. \\
\hline Radner 2015 (2) (31) & USA & To create a multimorbidity index based on health-related quality of life. \\
\hline Radner 2017 (36) & USA & $\begin{array}{l}\text { Determination of differences and factors contributing to the difference in patient global assessment of RA disease activity between RA patients with } \\
\text { multiple morbidities and those with RA only. }\end{array}$ \\
\hline Vial $2020(37)$ & France & Assessment of factors influencing the choice and effectiveness of DMARDs following failure of rituximab in RA (including co-existing conditions). \\
\hline Yoshihara $2016(38)$ & Japan & Investigation into whether RA patients have similar perioperative outcomes after elective joint replacement surgery, with multiple co-existing conditions. \\
\hline \multicolumn{3}{|l|}{ Case-control $(n=7)$} \\
\hline An 2019 (39) & USA & Investigating prevalence of comorbidities in RA vs non-RA controls and effect of comorbidities on health-related quality of life and healthcare expenditures. \\
\hline $\begin{array}{l}\text { Espiño-Lorenzo } 2013 \\
(26)\end{array}$ & Spain & $\begin{array}{l}\text { To determine whether RA patients prescribed biologic agents to control inflammatory activity have different comorbidity burden to those prescribed } \\
\text { conventional synthetic DMARDs; understand associations between comorbidity and other variables and between comorbidity and multimorbidity. }\end{array}$ \\
\hline Mikuls $2003(40)$ & USA & $\begin{array}{l}\text { Characterisation of self-reported functional disability and health related quality of life in RA cases, compared to controls from the same cohort, and } \\
\text { influence of other co-existing conditions and comorbidities on this. }\end{array}$ \\
\hline Panopoulos 2020 (22) & Greece & Comparison of prevalence of major comorbidities in age- and sex-matched case-control study of patients with APS, and patients with RA. \\
\hline Ramos 2019 (41) & Germany & $\begin{array}{l}\text { Investigation into the prevalence of selected comorbidities in patients with RA in comparison to age- and sex-matched patients without RA, and study the } \\
\text { association of comorbidity with rheumatologic care and self-reported RA outcomes. }\end{array}$ \\
\hline Ursum $2013(30)$ & The Netherlands & $\begin{array}{l}\text { Determination of prevalence of chronic comorbidities at onset of inflammatory arthritis and whether this is different from the number that one might } \\
\text { expect based on age and sex. }\end{array}$ \\
\hline Yoshida 2019 (42) & USA & Determination of the extent to which an extensive multimorbidity measure explained the excess mortality among RA patients. \\
\hline \multicolumn{3}{|l|}{ Cross-sectional $(\mathrm{n}=6)$} \\
\hline Lowe 2017 (28) & Australia & $\begin{array}{l}\text { To determine whether working-age adults with specific forms of musculoskeletal conditions are worse off in the presence of multimorbidity compared to } \\
\text { the rest of the musculoskeletal sample. }\end{array}$ \\
\hline Ma 2019 (43) & Malaysia & Establish additional data on the types and factors associated with drug-related problems in RA patients. \\
\hline
\end{tabular}




\begin{tabular}{|c|c|c|}
\hline Radner 2015 (3) (24) & USA & Description of treatment profile of multimorbid patients with RA, in contrast to patients with RA only. \\
\hline Shin 2019 (44) & South Korea & Association between socioeconomic status and comorbidity distribution among patients with RA. \\
\hline Tournadre 2019 (45) & France & Exploration of factors associated with fatigue in RA, focusing on social aspects, comorbidities, and treatment intake. \\
\hline Ziade 2020 (29) & Lebanon & $\begin{array}{l}\text { Evaluation of prevalence and pattern of comorbidities and risk factors in certain rheumatic diseases (including RA), and identify multimorbidity patterns and } \\
\text { evaluate gap between available screening recommendations and routine comorbidities' screening in daily practice. }\end{array}$ \\
\hline \multicolumn{3}{|c|}{ Randomised controlled trial: protocol $(n=1)$} \\
\hline Hider 2018 (16) & UK & $\begin{array}{l}\text { Evaluation of the feasibility and acceptability of a nurse-led integrated care review for people with inflammatory rheumatological conditions in primary } \\
\text { care, recognising the multimorbid nature of these patients. }\end{array}$ \\
\hline \multicolumn{3}{|l|}{ Systematic literature review $(n=3)$} \\
\hline Cutolo $2014(46)$ & Italy/ UK/ Netherlands & Evaluation of the physical and psychosocial extra-articular burden and comorbidities of treated RA and relationships among diverse disease manifestations. \\
\hline Gergianaki 2019 (27) & Greece & $\begin{array}{l}\text { Update of information regarding the comorbid state of rheumatic disease and COPD (prevalence, incidence), to examine whether patients with RD have } \\
\text { increased risk of developing COPD and vice versa, and implication of comorbidity on patient outcomes. }\end{array}$ \\
\hline $\begin{array}{l}\text { National Institute for } \\
\text { Clinical Excellence } \\
2003(20) \\
\end{array}$ & UK & Guidance on use of cyclo-oxygenase-II selective inhibitors for osteoarthritis and RA, taking comorbidities into account. \\
\hline \multicolumn{3}{|c|}{ Systematic literature review: protocol $(n=1)$} \\
\hline Canning $2020(15)$ & UK & Appraisal of literature to determine what is known on the effect, if any, of multimorbidity on mortality and other health-related outcomes in RA. \\
\hline \multicolumn{3}{|r|}{ (1: } \\
\hline Aslam $2018(47)$ & USA & Review of generic and specific comorbidity indices commonly used in RA research. \\
\hline Radner 2014 (2) & USA & $\begin{array}{l}\text { Review of conceptual differences between comorbidity and multimorbidity, underlines the importance of multimorbidity in a clinical context, and discusses } \\
\text { how this might impact care and research in rheumatology. }\end{array}$ \\
\hline Radner 2016 (48) & Austria & Review of multimorbidity in rheumatic diseases. \\
\hline Van Onna 2016 (49) & The Netherlands & Review of RA and ageing, and association with comorbidities. \\
\hline \multicolumn{3}{|l|}{ Editorial ( $\mathrm{n}=\mathbf{2})$} \\
\hline Lems 2017 (50) & The Netherlands & Investigation into cardiovascular comorbidities and acute events following a fragility fracture on background RA. \\
\hline Nikiphorou 2018 (25) & UK/Netherlands/Hungary & Summary of current work on comorbidities in rheumatic disease. \\
\hline \multicolumn{3}{|l|}{ Audit (n=1) } \\
\hline Daïen 2019 (51) & France & $\begin{array}{l}\text { To define the most common multimorbidities in chronic inflammatory rheumatic diseases, compare screening approach in clinic with EULAR } \\
\text { recommendations, validate points to consider for systematic standardized multimorbidity screening proposed by EULAR and assess feasibility of such } \\
\text { screening in clinic. }\end{array}$ \\
\hline \multicolumn{3}{|l|}{ Consensus statement $(n=1)$} \\
\hline Bernatsky 2013 (52) & Canada & $\begin{array}{l}\text { To develop best-practice consensus statements about the use of administrative data for rheumatic disease research, accounting for comorbidities } \\
\text { commonly encountered in this patient cohort. }\end{array}$ \\
\hline
\end{tabular}

Table 2: Summary of included records, by article type, with brief description of overview and aims of each article. RA= rheumatoid arthritis; DMARD=

disease-modifying anti-rheumatic drug; TNFi= tumour necrosis factor inhibitor; $\mathrm{RDCl}=$ rheumatic disease comorbidity index; $\mathrm{CVD}=$ cardiovascular disease;

APS = antiphospholipid syndrome; $C O P D=$ chronic obstructive pulmonary disease. 


\begin{tabular}{|c|c|c|c|}
\hline Author/Year & Definition of term? & Definition & $\begin{array}{l}\text { Is RA included as part of the term } \\
\text { comorbidity/multimorbidity? }\end{array}$ \\
\hline \multicolumn{4}{|l|}{ Comorbidity/ Co-morbidity } \\
\hline An 2019 (39) & No & N/A & No \\
\hline Aslam 2018 (47) & Yes & $\begin{array}{l}\text { Existence or occurrence of any additional entity during the clinical course of a } \\
\text { patient who has the index disease under study. }\end{array}$ & No \\
\hline Bernatsky 2013 (52) & No & N/A & No \\
\hline Bili $2011(34)$ & No & N/A & No \\
\hline Chen 2019 (18) & No & N/A & Yes \\
\hline Crilly 2014 (17) & Yes & $\begin{array}{l}\text { Any condition not classed as an extra-articular feature, as per Malmo } \\
\text { classification. }\end{array}$ & Yes, for extra-articular features \\
\hline Cutolo 2014 (46) & No & N/A & No \\
\hline Gil-Conesa 2020 (19) & No & N/A & Unclear \\
\hline Lems 2017 (50) & No & N/A & No \\
\hline Ma 2019 (43) & No & N/A & No \\
\hline Mikuls $2003(40)$ & No & N/A & No \\
\hline NICE 2003 (20) & No & N/A & Unclear \\
\hline Panopoulos $2020(22)$ & No & N/A & No \\
\hline Ramos 2019 (41) & No & N/A & No \\
\hline Shin $2019(44)$ & No & $\mathrm{N} / \mathrm{A}$ & No \\
\hline Ursum $2013(30)$ & Yes & $\begin{array}{l}\text { "Co-morbidity can be developed independently from IA, but it can also be the } \\
\text { result of IA treatment, common underlying causes and/or shared risk factors." }\end{array}$ & No \\
\hline Van Onna 2016 (49) & No & N/A & No \\
\hline Yoshihara $2016(38)$ & No & N/A & No \\
\hline \multicolumn{4}{|l|}{ Multimorbidity } \\
\hline Canning 2020 (15) & Yes & Co-existence of two or more long term conditions. & No \\
\hline Daïen 2019 (51) & Partly & $\begin{array}{l}\text { "The term multimorbidity is more appropriate than comorbidity in } \\
\text { inflammatory rheumatic diseases as the primary disease is unknown." }\end{array}$ & No \\
\hline Radner 2016 (48) & Yes & Co-existence of two or more chronic diseases in the same individual. & No \\
\hline Radner 2015 (1) (23) & Yes & Multimorbidity refers to a patient with multiple co-existing diseases. & No \\
\hline Radner 2015 (3) (24) & Yes & $\begin{array}{l}\text { "Multimorbidity takes into account all potential interactions of coexisting } \\
\text { diseases and its effect on patients' overall well-being." }\end{array}$ & No \\
\hline Radner 2015 (2) (31) & Yes & Holistic, patient-centred concept of co-existing conditions. & No \\
\hline
\end{tabular}




\begin{tabular}{|c|c|c|c|}
\hline Radner 2017 (36) & No & No formal definition given, but reference to counted multimorbidity index. & No \\
\hline Yoshida 2019 (42) & Yes & $\begin{array}{l}\text { Presence of two or more chronic conditions in an individual and representation } \\
\text { of an individual's accumulating morbidity burden. }\end{array}$ & No \\
\hline \multicolumn{4}{|l|}{ Comorbidity and Multimorbidity } \\
\hline Armagan $2018(32)$ & Partly & $\begin{array}{l}\text { In addition to RA, patients with at least } 1 \text { comorbidity were defined as } \\
\text { "comorbidity positive," others were "comorbidity negative." In addition to RA, } \\
\text { patients with at least } 2 \text { comorbidities were defined as multi-comorbidities. }\end{array}$ & No \\
\hline Biggioggero 2019 (33) & No & $\mathrm{N} / \mathrm{A}$ & No \\
\hline $\begin{array}{l}\text { Espiño-Lorenzo } 2013 \\
(26)\end{array}$ & Yes & $\begin{array}{l}\text { Multimorbidity: coincidence of two or more diseases in a patient with RA. } \\
\text { Comorbidity: as per Charlson index }\end{array}$ & No \\
\hline Gergianaki 2019 (27) & Partly & $\begin{array}{l}\text { Multimorbidity: coexistence of at least two diseases or psychosocial/somatic } \\
\text { risk factors. Co-morbidity not defined. }\end{array}$ & No \\
\hline Hider 2018 (16) & Partly & $\begin{array}{l}\text { Multimorbidity: co-occurrence of two or more long-term conditions. Co- } \\
\text { morbidity: not formally defined. }\end{array}$ & No \\
\hline Hope 2020 (35) & No & N/A & No \\
\hline Lowe 2017 (28) & Yes & $\begin{array}{l}\text { "Chronic co-occurring conditions are termed multimorbidity, or in the context } \\
\text { of a primary condition, comorbidity." }\end{array}$ & No \\
\hline Nikiphorou 2018 (25) & Yes & $\begin{array}{l}\text { Multimorbidity: coexistence of two or more chronic diseases in the same } \\
\text { individual. Comorbidity: co-occurrence of any distinct additional entities. }\end{array}$ & No \\
\hline Radner 2014 (2) & Yes & $\begin{array}{l}\text { Comorbidity focuses on the index disease and addresses the co-occurrence of } \\
\text { any distinct additional entities. Multimorbidity can be defined as "coexistence } \\
\text { of two or more chronic diseases in the same individual." }\end{array}$ & No \\
\hline Tournadre 2019 (45) & No & No formal definition, Multimorbidity Score is referenced. & No \\
\hline Vial 2020 (37) & No & N/A & No \\
\hline Ziade 2020 (29) & Yes & $\begin{array}{l}\text { Multimorbidity: concurrent presence of more than one non-communicable } \\
\text { disease. Co-morbidity: non-communicable disease and conditions associated } \\
\text { with it. }\end{array}$ & No \\
\hline \multicolumn{4}{|l|}{ No specific term used } \\
\hline Daïen 2018 (21) & No & N/A (see text for details) & No \\
\hline
\end{tabular}

Table 3: Summary of terms used in included articles to define the presence of co-existing conditions, and, where applicable, the definition given within the main body of text. $R A=$ rheumatoid arthritis; $I A=$ inflammatory arthritis 


\begin{tabular}{|c|c|c|}
\hline Author/Year & Comorbidity/multimorbidity Index used & Context of use for comorbidity/multimorbidity index \\
\hline Biggioggero 2019 (33) & $\mathrm{RDCl}$ & $\begin{array}{l}\text { Predictors of treatment choice, clinical response, and retention rate in RA } \\
\text { patients treated with a first-line TNFi. }\end{array}$ \\
\hline Crilly 2014 (17) & Malmo classification (for extra-articular features) & $\begin{array}{l}\text { Explore associations between comorbidities and extra-articular features with } \\
\text { QOL in patients with RA. }\end{array}$ \\
\hline $\begin{array}{l}\text { Espiño-Lorenzo } 2013 \\
(26)\end{array}$ & Charlson Index & $\begin{array}{l}\text { Assess difference in comorbidity burden between RA patients prescribed } \\
\text { biologics and those prescribed conventional synthetic DMARDs. }\end{array}$ \\
\hline Radner 2015 (1) (23) & cMMI & Predictors of remission or low disease activity. \\
\hline Radner 2015 (3) (24) & cMMI & $\begin{array}{l}\text { Assess effect of multimorbidity on treatment status, accounting for factors } \\
\text { that affect treatment decisions. }\end{array}$ \\
\hline Radner 2015 (2) (31) & $\begin{array}{l}\text { Development of two new indices, using count and } \\
\text { weighted count of co-morbid conditions, forming the MMI }\end{array}$ & Develop MMI based on HrQOL. \\
\hline Radner 2017 (36) & cMMI & $\begin{array}{l}\text { Explore patient global assessment of disease activity in patients with RA and } \\
\text { multimorbidity, and RA only. }\end{array}$ \\
\hline Ramos 2019 (41) & Based on Elixhauser Comorbidity Index & $\begin{array}{l}\text { Investigate prevalence of comorbidities in persons with RA, compared to age- } \\
\text { and sex-matched persons without RA; association of comorbidity with } \\
\text { rheumatologic care and with self-reported RA outcomes. }\end{array}$ \\
\hline Tournadre 2019 (45) & $\begin{array}{l}\text { MMI comprising two indices, using count and weighted } \\
\text { count of co-morbid conditions }\end{array}$ & Factors associated with fatigue in RA, including co-exisiting conditions. \\
\hline Vial 2020 (37) & $\begin{array}{l}\text { MMI comprising two indices, using count and weighted } \\
\text { count of co-morbid conditions }\end{array}$ & $\begin{array}{l}\text { Predictors of choice of biologic DMARD after rituximab failure in RA patients; } \\
\text { analysis of effectiveness of these biologics, taking into account comorbidities. }\end{array}$ \\
\hline Yoshida 2019 (42) & wMMI & $\begin{array}{l}\text { Explore association between post-diagnosis multimorbidity and lifestyle } \\
\text { changes with excess mortality of RA }\end{array}$ \\
\hline Yoshihara 2016 (38) & Elixhauser comorbidity index & $\begin{array}{l}\text { Comparison of outcomes of joint replacement surgery between patients with } \\
\text { and without RA, controlling for comorbidities. }\end{array}$ \\
\hline
\end{tabular}

Table 4: Summary of articles using a comorbidity/multimorbidity index score, and the context for the use of this score. RDCl= rheumatic diseases

comorbidity index; $\mathrm{MMI}=$ multimorbidity index; $\mathrm{CMMI}=$ counted multimorbidity index; $\mathrm{WMMl}=$ weighted multimorbidity index. $\mathrm{RA}=$ rheumatoid arthritis; $\mathrm{TNFi}=$ tumour necrosis factor inhibitors; $\mathrm{QOL}=$ quality of life; DMARDs= disease-modifying anti-rheumatic drugs; HrQOL= health-related quality of life. 


\section{References}

1. N'Goran AA, Blaser J, Deruaz-Luyet A, Senn N, Frey P, Haller DM, et al. From chronic conditions to relevance in multimorbidity: $A$ four-step study in family medicine. Fam Pract [Internet]. 2016 Aug 1 [cited 2020 Sep 2];33(4):439-44. Available from: https://academic.oup.com/fampra/article/33/4/439/1749443

2. Radner H, Yoshida K, Smolen JS. Multimorbidity and rheumatic conditions - Enhancing the concept of comorbidity. Nat Rev Rheumatol [Internet]. 2014;10(4):252-6. Available from: http://www.nature.com/nrrheum/archive/index.html

3. NIHR Strategic Framework for Multiple Long-Term Conditions (Multimorbidity) MLTC-M Research [Internet]. [cited 2020 Oct 6]. Available from:

https://www.nihr.ac.uk/documents/research-on-multiple-long-term-conditionsmultimorbidity-mltc-m/24639

4. Multimorbidity | Topics A to Z | CKS | NICE [Internet]. [cited 2020 Oct 7]. Available from: https://cks.nice.org.uk/topics/multimorbidity/

5. Feinstein AR. The pre-therapeutic classification of co-morbidity in chronic disease. J Chronic Dis. 1970 Dec 1;23(7):455-68.

6. Nikiphorou E, Norton S, Carpenter L, Dixey J, Andrew Walsh D, Kiely P, et al. Secular Changes in Clinical Features at Presentation of Rheumatoid Arthritis: Increase in Comorbidity But Improved Inflammatory States. Arthritis Care Res [Internet]. 2017 Jan 1 [cited 2020 Dec 4];69(1):21-7. Available from: https://pubmed.ncbi.nlm.nih.gov/27564223/

7. Nikiphorou E, Nurmohamed MT, Szekanecz Z. Editorial: Comorbidity burden in rheumatic diseases [Internet]. Vol. 5, Frontiers in Medicine. Frontiers Media S.A.; 2018 [cited 2020 Dec 4]. Available from: https://www.ncbi.nlm.nih.gov/pmc/articles/PMC6037715/

8. Nikiphorou E, De Lusignan S, Mallen C, Roberts J, Khavandi K, Bedarida G, et al. Prognostic value of comorbidity indices and lung diseases in early rheumatoid arthritis: A UK populationbased study. Rheumatol (United Kingdom) [Internet]. 2020 Jun 1 [cited 2020 Dec 4];59(6):1296-305. Available from: https://pubmed.ncbi.nlm.nih.gov/31580449/

9. Fortin M, Lapointe L, Hudon C, Vanasse A. Multimorbidity is common to family practice: is it commonly researched? [Internet]. Vol. 51, Canadian family physician Médecin de famille canadien. College of Family Physicians of Canada; 2005 [cited 2020 Sep 2]. p. 244-5. Available from: www.cfpc.ca/cfp

10. Barnett K, Mercer SW, Norbury M, Watt G, Wyke S, Guthrie B. Epidemiology of multimorbidity and implications for health care, research, and medical education: A crosssectional study. Lancet. 2012 Jul 7;380(9836):37-43.

11. Cochrane Handbook for Systematic Reviews of Interventions | Cochrane Training [Internet]. [cited 2020 Aug 31]. Available from: https://training.cochrane.org/handbook/current

12. Moher D, Liberati A, Tetzlaff J, Altman DG, Altman D, Antes G, et al. Preferred reporting items for systematic reviews and meta-analyses: The PRISMA statement. Vol. 6, PLoS Medicine. 2009.

13. Browse the Registry - Research Registry [Internet]. [cited 2020 Oct 7]. Available from: https://www.researchregistry.com/browse-the-registry\#registryofsystematicreviewsmetaanalyses/registryofsystematicreviewsmeta-analysesdetails/5f6622d6faab730017d6cd20/ 
14. 2.6.2 Review question - JBI Manual for Evidence Synthesis - JBI GLOBAL WIKI [Internet]. [cited 2021 Jan 29]. Available from: https://wiki.jbi.global/display/MANUAL/2.6.2+Review+question

15. Canning J, Siebert S, Jani BD, Mair FS, Nicholl BI. Examining the relationship between rheumatoid arthritis, multimorbidity and adverse health-related outcomes: A systematic review protocol. J comorbidity [Internet]. 2020;10:2235042X20906657. Available from: http://ovidsp.ovid.com/ovidweb.cgi?T=JS\&PAGE=reference\&D=prem\&NEWS=N\&AN=322066 33

16. Hider SL, Bucknall M, Cooke K, Cooke K, Finney AG, Goddin D, et al. The INCLUDE study: INtegrating and improving Care for patients with infLammatory rheUmatological DisordErs in the community; identifying multimorbidity: Protocol for a pilot randomized controlled trial. J comorbidity [Internet]. 2018;8(1):2235042X18792373. Available from: http://ovidsp.ovid.com/ovidweb.cgi?T=JS\&PAGE=reference\&D=prem2\&NEWS=N\&AN=30191 145

17. Crilly MA, Johnston MC, Black C. Relationship of EQ-5D quality of life with the presence of comorbidity and extra-articular features in patients with rheumatoid arthritis. Qual Life Res [Internet]. 2014;23(5):1435-43. Available from:

http://ovidsp.ovid.com/ovidweb.cgi?T=JS\&PAGE=reference\&D=med11\&NEWS=N\&AN=24322 906

18. Chen K-L, Chiu H-Y, Lin J-H, Ye J-D, Cho Y-H, Li K-J, et al. Prevalence, clinical features and treatment pattern of patients with concurrent diagnoses of rheumatoid arthritis and psoriatic disease: results of a 14-year retrospective study in a tertiary referral center. Ther Adv Chronic Dis [Internet]. 2019;10:2040622319847900. Available from:

http://ovidsp.ovid.com/ovidweb.cgi?T=JS\&PAGE=reference\&D=prem3\&NEWS=N\&AN=31205 646

19. Gil-Conesa M, Del-Moral-Luque JA, Gil-Prieto R, Gil-de-Miguel A, Mazzuccheli-Esteban R, Rodriguez-Caravaca G. Hospitalization burden and comorbidities of patients with rheumatoid arthritis in Spain during the period 2002-2017. BMC Health Serv Res [Internet].

2020;20(1):374. Available from:

http://ovidsp.ovid.com/ovidweb.cgi?T=JS\&PAGE=reference \&D=prem\&NEWS=N\&AN=323662 47

20. National Institute of Clinical Excellence. Guidance on the use of cyclo-oxygenase (Cox) II selective inhibitors, celecoxib, rofecoxib, meloxicam and etodolac for osteoarthritis and rheumatoid arthritis. National Institute for Clinical Excellence: National Institute for Clinical Excellence (NICE); 2003.

21. Daien Cl, Tubery A, Cailar G du, Mura T, Roubille F, Morel J, et al. Application of the 2015/2016 EULAR recommendations for cardiovascular risk in daily practice: data from an observational study. Ann Rheum Dis [Internet]. 2018;77(4):625-6. Available from: http://ovidsp.ovid.com/ovidweb.cgi?T=JS\&PAGE=reference\&D=med15\&NEWS=N\&AN=28478 400

22. Panopoulos S, Thomas K, Georgiopoulos G, Boumpas D, Katsiari C, Bertsias G, et al. Comparable or higher prevalence of comorbidities in antiphospholipid syndrome vs rheumatoid arthritis: a multicenter, case-control study. Rheumatology (Oxford) [Internet]. 2020; Available from:

http://ovidsp.ovid.com/ovidweb.cgi?T=JS\&PAGE=reference\&D=medp\&NEWS=N\&AN=325967 27

23. Radner H, Yoshida K, Frits M, lannaccone C, Shadick NA, Weinblatt M, et al. The impact of 
multimorbidity status on treatment response in rheumatoid arthritis patients initiating disease-modifying anti-rheumatic drugs. Rheumatology (Oxford) [Internet].

2015;54(11):2076-84. Available from:

http://ovidsp.ovid.com/ovidweb.cgi?T=JS\&PAGE=reference\&D=med12\&NEWS=N\&AN=26163 688

24. Radner H, Yoshida K, Hmamouchi I, Dougados M, Smolen JS, Solomon DH. Treatment Patterns of Multimorbid Patients with Rheumatoid Arthritis: Results from an International Cross-sectional Study. J Rheumatol [Internet]. 2015;42(7):1099-104. Available from: http://ovidsp.ovid.com/ovidweb.cgi?T=JS\&PAGE=reference \&D=med12\&NEWS=N\&AN=26034 147

25. Nikiphorou E, Nurmohamed MT, Szekanecz Z. Editorial: Comorbidity Burden in Rheumatic Diseases. Front Med [Internet]. 2018;5:197. Available from:

http://ovidsp.ovid.com/ovidweb.cgi?T=JS\&PAGE=reference\&D=prem2\&NEWS=N\&AN=30018 957

26. Espino-Lorenzo P, Manrique-Arija S, Urena I, Jimenez-Nunez FG, Lopez-Lasanta M, RomeroBarco CM, et al. Baseline comorbidities in patients with rheumatoid arthritis who have been prescribed biological therapy: a case control study. Reumatol Clin [Internet]. 2013;9(1):1823. Available from:

http://ovidsp.ovid.com/ovidweb.cgi?T=JS\&PAGE=reference \&D=med10\&NEWS=N\&AN=22938 792

27. Gergianaki I, Tsiligianni I. Chronic obstructive pulmonary disease and rheumatic diseases: A systematic review on a neglected comorbidity. J comorbidity [Internet].

2019;9:2235042X18820209. Available from: http://ovidsp.ovid.com/ovidweb.cgi?T=JS\&PAGE=reference\&D=prem3\&NEWS=N\&AN=31309 081

28. Lowe D, Taylor M. Associations between multimorbidity and additional burden for workingage adults with specific forms of musculoskeletal conditions: a cross-sectional study. BMC Musculoskelet Disord [Internet]. 2017;18(1):135. Available from: http://www.biomedcentral.com/bmcmusculoskeletdisord/

29. Ziade N, El Khoury B, Zoghbi M, Merheb G, Abi Karam G, Mroue' K, et al. Prevalence and pattern of comorbidities in chronic rheumatic and musculoskeletal diseases: the COMORD study. Sci Rep [Internet]. 2020;10(1):7683. Available from:

http://ovidsp.ovid.com/ovidweb.cgi?T=JS\&PAGE=reference\&D=prem\&NEWS=N\&AN=323768 50

30. Ursum J, J.C. K, Twisk JWR, Peters MJL, Schellevis FG, Nurmohamed MT. Prevalence of chronic diseases at the onset of inflammatory arthritis: A population-based study. Fam Pract [Internet]. 2013;30(6):615-20. Available from: http://ovidsp.ovid.com/ovidweb.cgi?T=JS\&PAGE=reference\&D=emed14\&NEWS=N\&AN=3703 30712

31. Radner H, Yoshida K, Mjaavatten MD, Aletaha D, Frits M, Lu B, et al. Development of a multimorbidity index: Impact on quality of life using a rheumatoid arthritis cohort. Semin Arthritis Rheum [Internet]. 2015;45(2):167-73. Available from:

http://ovidsp.ovid.com/ovidweb.cgi?T=JS\&PAGE=reference\&D=med12\&NEWS=N\&AN=26209 213

32. Armagan B, Sari A, Erden A, Kilic L, Erdat EC, Kilickap S, et al. Starting of biological disease modifying antirheumatic drugs may be postponed in rheumatoid arthritis patients with 
multimorbidity: Single center real life results. Medicine (Baltimore) [Internet].

2018;97(13):e9930. Available from:

http://ovidsp.ovid.com/ovidweb.cgi?T=JS\&PAGE=reference\&D=med15\&NEWS=N\&AN=29595 700

33. Biggioggero M, Mesina F, Favalli EG. The Use of Rheumatic Disease Comorbidity Index for Predicting Clinical Response and Retention Rate in a Cohort of Rheumatoid Arthritis Patients Receiving Tumor Necrosis Factor Alpha Inhibitors. Biomed Res Int [Internet]. 2019;2019:6107217. Available from:

http://ovidsp.ovid.com/ovidweb.cgi?T=JS\&PAGE=reference \&D=med16\&NEWS=N\&AN=30733 963

34. Bili A, Schroeder LL, Ledwich L, Kirchner HL, Newman ED, Wasko MCM. Patterns of preventive health services in rheumatoid arthritis patients compared to a primary care patient population. Rheumatol Int [Internet]. 2011;31(9):1159-65. Available from: http://ovidsp.ovid.com/ovidweb.cgi?T=JS\&PAGE=reference\&D=med8\&NEWS=N\&AN=203490 66

35. Hope HF, Hyrich KL, Anderson J, Bluett J, Sergeant JC, Barton A, et al. The predictors of and reasons for non-adherence in an observational cohort of patients with rheumatoid arthritis commencing methotrexate. Adebajo A Al-Ansari A, Amarasena R, Bukhari M, Callan M, Chelliah EG, Chinoy H, Cooper A, Dasgupta B, Davis M, Galloway J, Gough A, Green M, Gullick N, Hamilton J, Hassan W, Hider S, Hyrich K, Kamath S, Knight S, Lane S, Lee M, Levy S, Macphie L, Marguer AK, editor. Rheumatology (Oxford) [Internet]. 2020;59(1):213-23. Available from:

http://ovidsp.ovid.com/ovidweb.cgi?T=JS\&PAGE=reference\&D=medl\&NEWS=N\&AN=313026 92

36. Radner H, Yoshida K, Tedeschi S, Studenic P, Frits M, lannaccone C, et al. Different Rating of Global Rheumatoid Arthritis Disease Activity in Rheumatoid Arthritis Patients With Multiple Morbidities. Arthritis Rheumatol (Hoboken, NJ) [Internet]. 2017;69(4):720-7. Available from: http://ovidsp.ovid.com/ovidweb.cgi?T=JS\&PAGE=reference\&D=med14\&NEWS=N\&AN=27860 467

37. Vial G, De Pouilly A, Scouppe L, Pereira B, Daien C, Lukas C, et al. Factors influencing the choice of biologic therapy following Rituximab in patients with rheumatoid arthritis: $A$ retrospective study using propensity score. Jt bone spine [Internet]. 2020;87(1):43-8. Available from:

http://ovidsp.ovid.com/ovidweb.cgi?T=JS\&PAGE=reference\&D=prem\&NEWS=N\&AN=313698 68

38. Yoshihara H, Yoneoka D, Margalit A, Zuckerman JD. Rheumatoid arthritis patients undergoing total hip and knee arthroplasty have better in-hospital outcomes compared with nonrheumatoid arthritis patients. Clin Exp Rheumatol [Internet]. 2016;34(2):270-5. Available from:

http://ovidsp.ovid.com/ovidweb.cgi?T=JS\&PAGE=reference \&D=med13\&NEWS=N\&AN=26886 847

39. An J, Nyarko E, Hamad MA. Prevalence of comorbidities and their associations with healthrelated quality of life and healthcare expenditures in patients with rheumatoid arthritis. Clin Rheumatol [Internet]. 2019;38(10):2717-26. Available from:

http://ovidsp.ovid.com/ovidweb.cgi?T=JS\&PAGE=reference\&D=medI\&NEWS=N\&AN=311345 04

40. Mikuls T, Saag K, Criswell L, Merlino L, Cerhan JR. Health related quality of life in women with 
elderly onset rheumatoid arthritis. J Rheumatol [Internet]. 2003;30(5):952-7. Available from: http://ovidsp.ovid.com/ovidweb.cgi?T=JS\&PAGE=reference\&D=med5\&NEWS=N\&AN=127348 88

41. Luque Ramos A, Redeker I, Hoffmann F, Callhoff J, Zink A, Albrecht K. Comorbidities in Patients with Rheumatoid Arthritis and Their Association with Patient-reported Outcomes: Results of Claims Data Linked to Questionnaire Survey. J Rheumatol [Internet]. 2019;46(6):564-71. Available from:

http://ovidsp.ovid.com/ovidweb.cgi?T=JS\&PAGE=reference\&D=prem\&NEWS=N\&AN=306471 70

42. Yoshida K, Lin T-C, Wei M, Malspeis S, Chu SH, Camargo CA, et al. The roles of post-diagnosis accumulation of morbidities and lifestyle changes on excess total and cause-specific mortality risk in rheumatoid arthritis. Arthritis Care Res (Hoboken) [Internet]. 2019; Available from: http://ovidsp.ovid.com/ovidweb.cgi?T=JS\&PAGE=reference\&D=medp\&NEWS=N\&AN=318117 08

43. Ma SN, Zaman Huri H, Yahya F. Drug-related problems in patients with rheumatoid arthritis. Ther Clin Risk Manag [Internet]. 2019;15:505-24. Available from: http://ovidsp.ovid.com/ovidweb.cgi?T=JS\&PAGE=reference\&D=prem3\&NEWS=N\&AN=30962 689

44. Shin A, Shin S, Kim J, Ha Y-J, Lee Y, Song Y. Association between socioeconomic status and comorbidities among patients with rheumatoid arthritis: Results of a nationwide crosssectional survey. Rheumatol (United Kingdom) [Internet]. 2019;58(9):1617-22. Available from: http://rheumatology.oxfordjournals.org/

45. Tournadre A, Pereira B, Gossec L, Soubrier M, Dougados M. Impact of comorbidities on fatigue in rheumatoid arthritis patients: results from a nurse-led program for comorbidities management (COMEDRA). Joint, bone, spine [Internet]. 2019;86(1):55-60. Available from: https://www.cochranelibrary.com/central/doi/10.1002/central/CN-02084288/full

46. Cutolo M, Kitas GD, van Riel PLCM. Burden of disease in treated rheumatoid arthritis patients: going beyond the joint. Semin Arthritis Rheum [Internet]. 2014;43(4):479-88. Available from: http://ovidsp.ovid.com/ovidweb.cgi?T=JS\&PAGE=reference\&D=med11\&NEWS=N\&AN=24080 116

47. Aslam F, Khan NA. Tools for the Assessment of Comorbidity Burden in Rheumatoid Arthritis. Front Med [Internet]. 2018;5:39. Available from:

http://ovidsp.ovid.com/ovidweb.cgi?T=JS\&PAGE=reference\&D=prem2\&NEWS=N\&AN=29503 820

48. Radner H. Multimorbidity in rheumatic conditions. Wien Klin Wochenschr [Internet]. 2016;128(21-22):786-90. Available from: http://ovidsp.ovid.com/ovidweb.cgi?T=JS\&PAGE=reference\&D=med13\&NEWS=N\&AN=27738 754

49. van Onna M, Boonen $A$. The challenging interplay between rheumatoid arthritis, ageing and comorbidities. BMC Musculoskelet Disord [Internet]. 2016;17:184. Available from: http://ovidsp.ovid.com/ovidweb.cgi?T=JS\&PAGE=reference\&D=med13\&NEWS=N\&AN=27118 031

50. Lems WF. Is Fragility Fracture a Strong Risk Factor for a Cardiovascular Event in Rheumatoid Arthritis? The Challenge of Dealing with Multiple Comorbidities. J Rheumatol [Internet]. 2017;44(5):545-6. Available from:

http://ovidsp.ovid.com/ovidweb.cgi?T=JS\&PAGE=reference\&D=med14\&NEWS=N\&AN=28461 
515

51. Daien $\mathrm{Cl}$, Tubery A, Beurai-Weber M, du Cailar G, Picot M-C, Jaussent A, et al. Relevance and feasibility of a systematic screening of multimorbidities in patients with chronic inflammatory rheumatic diseases. Jt bone spine [Internet]. 2019;86(1):49-54. Available from:

http://ovidsp.ovid.com/ovidweb.cgi?T=JS\&PAGE=reference\&D=medl\&NEWS=N\&AN=296549 49

52. Bernatsky S, Lix L, O'Donnell S. Consensus statements for the use of administrative health data in rheumatic disease research and surveillance. J Rheumatol [Internet]. 2013;40(1):6673. Available from: http://www.jrheum.org/content/40/1/66.full.pdf+html 


\section{Figure legends}

Figure 1a (left panel): Comorbidity in rheumatoid arthritis. Rheumatoid arthritis is the index disease, and all other conditions co-exist, and are treated as, separate secondary entities, often by other specialties. Conditions such as anxiety and depression may be easily missed unless specifically screened for (indicated by broken arrow). Co-existing conditions may also arise as a result of treatment, of both the index and co-existing diseases, but also may be missed, or classified as entities in their own right.

Figure 1b (right panel): Multimorbidity in rheumatoid arthritis. The focus is on the patients, and all conditions co-exist equally. Treatment and outcomes are addressed in a holistic manner, keeping the patient at the centre of all assessment and decision-making. 